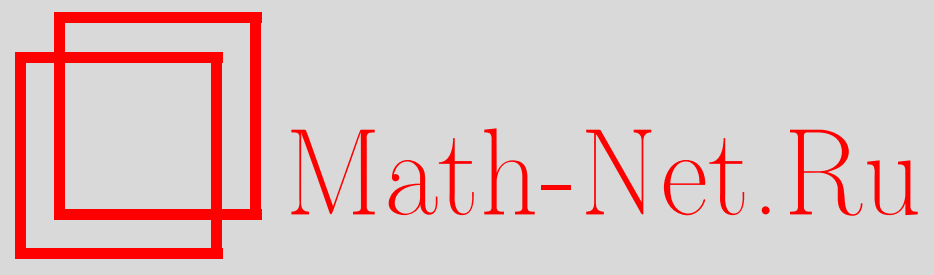

E. Baurdoux, A. Kyprianou, The Shepp-Shiryaev Stochastic Game Driven by a Spectrally Negative Lévy Process, Теория вероятн. и ее примен., 2008, том 53, выпуск 3, 588-609

DOI: https://doi.org/10.4213/tvp2453

Использование Общероссийского математического портала Math-Net.Ru подразумевает, что вы прочитали и согласны с пользовательским соглашением

http://www . mathnet.ru/rus/agreement

Параметры загрузки:

IP : 54.92 .164 .108

26 апреля 2023 г., 13:49:58

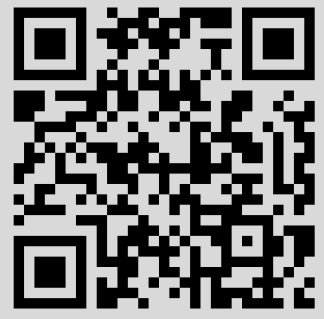




\section{THE SHEPP-SHIRYAEV STOCHASTIC GAME DRIVEN BY A SPECTRALLY NEGATIVE LÉVY PROCESS}

В [15] был рассмотрен стохастический игровой аналог задачи об оптимальной остановке Шеппа-Ширяева (ср. с [23] и [24]) для экспоненциального броуновского движения. Мы рассматриваем ту же стохастическую игру (которую называем стохастической игрой Шеппа-Ширяева), но для спектрально-отрицательного процесса Леви и для более широкого класса параметров. В отличие от [15], в настоящей статье методы стохастического анализа не являются преобладаюшими. Это, главным образом, связано с тем, что для предполагаемых решений трудно получить вариационные неравенства и приходится работать с нелокальными интегродифференциальными операторами. Взамен мы используем разнообразную технику, в том числе теорию флуктуаций, методы стохастического анализа, связанные с мартингальной характеризацией, и сведение стохастической игры к задаче об оптимальной остановке.

Ключевые слова и фразы: стохастические игры, оптимальная остановка, принципы склеивания, флуктуационные теоремы, процессы Леви.

1. Introduction. Let $X=\left\{X_{t}: t \geqslant 0\right\}$ be a Lévy process defined on a filtered probability space $(\Omega, \mathscr{F}, \mathbb{F}, \mathbf{P})$, where $\mathbb{F}=\left\{\mathscr{F}_{t}: t \geqslant 0\right\}$ is the filtration generated by $X$ which is naturally enlarged (cf. [5, Definition 1.3.38]). For $x \in \mathbf{R}$, denote by $\mathbf{P}_{x}$ the law of $X$ when it is started at $x$ and write simply $\mathbf{P}_{0}=\mathbf{P}$. Accordingly, we shall write $\mathbf{E}_{x}$ and $\mathbf{E}$ for the associated expectation operators. We shall assume throughout that $X$ is spectrally negative, meaning here that it has no positive jumps and that it is not the negative of a subordinator. It is well known that the latter allows us to talk about the Laplace exponent $\psi(\theta):=\ln \mathbf{E}\left[e^{\theta X_{1}}\right]$ for $\theta \geqslant 0$, which will be of frequent use in the sequel. The Laplace exponent necessarily takes the form

$$
\psi(\theta)=a \theta+\frac{1}{2} \sigma^{2} \theta^{2}+\int_{(-\infty, 0)}\left(e^{\theta x}-1-x \theta \mathbf{1}_{\{x>-1\}}\right) \Pi_{X}(d x),
$$

* Department of Statistics, London School of Economics, Houghton Street London, WC2A 2AE, England; e-mail: e.j.baurdoux@lse.ac.uk

** Department of Mathematical Sciences, University of Bath, Claverton Down, Bath, BA2 7AY, England; e-mail: a.kyprianou@bath.ac.uk 
where $a \in \mathbf{R}, \sigma \geqslant 0$ is the Gaussian coefficient and $\Pi_{X}$ is a measure concentrated on $(-\infty, 0)$ satisfying $\int_{(-\infty, 0)}\left(1 \wedge x^{2}\right) \Pi_{X}(d x)<\infty$. The reader is referred to [4] for a complete introduction to the theory of Lévy processes.

Denote by $\mathscr{T}_{0, \infty}$ the family of all $[0, \infty]$-valued stopping times with respect to $\mathbb{F}$. We are interested in establishing a solution to a special class of stochastic games which are driven by spectrally negative Lévy processes. Specifically, for a given $q>0$ and $\delta>0$, we study the stochastic game consisting of two players and expected pay-off given by

$$
M_{x}(\tau, \sigma):=\mathbf{E}_{x}\left[e^{-q \tau+\left(x \vee \bar{X}_{\tau}\right)} \mathbf{1}_{\{\tau \leqslant \sigma, \tau<\infty\}}+e^{-q \sigma}\left(e^{x \vee \bar{X}_{\sigma}}+\delta e^{X_{\sigma}}\right) \mathbf{1}_{\{\sigma<\tau\}}\right]
$$

for $x \geqslant 0$, where $\bar{X}_{t}=\sup _{s \leqslant t} X_{s}$ denotes the running supremum of $X$ and where $a \vee b=\max (a, b)$. The inf-player's objective is to choose some $\sigma \in \mathscr{T}_{0, \infty}$ which minimizes (1), whereas the sup-player chooses some $\tau \in \mathscr{T}_{0, \infty}$ which maximizes this quantity. Our aim is to prove the existence of a saddle point $\left(\tau^{*}, \sigma^{*}\right)$ such that

$$
M_{x}\left(\tau, \sigma^{*}\right) \leqslant M_{x}\left(\tau^{*}, \sigma^{*}\right) \leqslant M_{x}\left(\tau^{*}, \sigma\right)
$$

for all $\tau, \sigma \in \mathscr{T}_{0, \infty}$ and for all $x \geqslant 0$. The pair $\left(\tau^{*}, \sigma^{*}\right)$ is also known as a Nash equilibrium (cf. [9] and [20]). When such a pair of stopping times exists, we say that it solves the stochastic game (1) and we denote the corresponding value by

$$
V(x)=M_{x}\left(\tau^{*}, \sigma^{*}\right) \quad \text { for } \quad x \geqslant 0 .
$$

Note that we have included the indicator $\mathbf{1}_{\{\tau<\infty\}}$ in $(1)$, since $e^{-q t+\left(x \vee \bar{X}_{t}\right)}$ may not be well defined for $t=\infty$.

When $q=0$, this issue does not occur, since $e^{x \vee \bar{X}_{t}}$ is monotone in $t$, and in this case we are interested in the saddle point to the stochastic game which, for a given $\delta>0$, has expected pay-off given by

$$
M_{x}(\tau, \sigma)=\mathbf{E}_{x}\left[e^{x \vee \bar{X}_{\tau}} \mathbf{1}_{\{\tau \leqslant \sigma\}}+\left(e^{x \vee \bar{X}_{\sigma}}+\delta e^{X_{\sigma}}\right) \mathbf{1}_{\{\sigma<\tau\}}\right] .
$$

The game (1) was solved for $q>\psi(1) \geqslant 0$ (under an extra technical assumption on the parameters) for a Brownian motion in [15]. In some sense, that case is easier, since for a Brownian motion we can use standard Itô calculus and general theory of optimal stopping to show that a solution to a related free boundary problem (with a differential operator) also solves the game (1). The solution to this free boundary problem is readily found in terms of exponential functions. For a Lévy process with jumps, the corresponding free boundary problem seems more difficult to solve directly (or even to establish existence of a solution), as it involves an integro-differential operator. Instead, we use a mixture of fluctuation theory, martingale techniques and reduction of the stochastic game to an optimal stopping problem to solve (1). As a by-product, we find that a technical assumption in [15] is not needed, see Remark 5 . 
When $\psi(1)=q>0$, the stochastic game (1) can be understood to characterize the risk-neutral price of a so-called game option in a simple market consisting of a risky asset the value of which is given by $\left\{e^{X_{t}}: t \geqslant 0\right\}$ and a riskless asset which grows at rate $q$ (cf. [12]). The latter game option is an American-type contract with infinite horizon which offers the holder the right but not the obligation to claim $e^{x \backslash \bar{X}_{\tau}}$ at any stopping time $\tau \in \mathscr{T}_{0, \infty}$, but in addition, the contract also gives the writer the right but not the obligation to force a payment of $e^{x \vee \bar{X}_{\sigma}}+\delta e^{X_{\sigma}}$ at any stopping time $\sigma \in \mathscr{T}_{0, \infty}$; that is, what the holder would claim at that moment plus a penalty proportional to the current value of the asset. However, in this paper we do not discuss the relevance of the stochastic games (1) and (2) in the context of mathematical finance.

The stochastic games (1) and (2) are closely related to the SheppShiryaev optimal stopping problem

$$
U(x)=\sup _{\tau \in \mathscr{T}_{0, \infty}} \mathbf{E}\left[e^{-q \tau+\left(x \vee \bar{X}_{\tau}\right)} \mathbf{1}_{\{\tau<\infty\}}\right]
$$

which characterizes the value of a perpetual Russian option (cf. [23] and [24]) in the Brownian case and [2] for the Lévy case). See also [6], [8], and [18] for the finite expiry case and [11] for a linear programming approach. Indeed, if it is the case that the stochastic saddle point in (1) is achieved at $\sigma=\infty$, then it holds that $U=V$. In the article [24], an idea which is instrumental in helping provide the solution to (3), is to change measure from $\mathbf{P}$ to $\mathbf{P}^{1}$, where

$$
\left.\frac{d \mathbf{P}^{\lambda}}{d \mathbf{P}}\right|_{\mathscr{F}_{t}}=e^{\lambda X_{t}-\psi(\lambda) t}
$$

defines an equivalent measure on $\left\{\mathscr{F}_{t}: t \geqslant 0\right\}$ for any $\lambda \geqslant 0$. Under $\mathbf{P}^{\lambda}$, the process $X$ still belongs to the class of spectrally negative processes and its Laplace exponent is given by

$$
\psi_{\lambda}(\theta)=\psi(\theta+\lambda)-\psi(\lambda) \quad \text { for } \quad \theta \geqslant-\lambda .
$$

(For the latter conclusion it is important that $\mathbb{F}$ is naturally enlarged as opposed to satisfying the usual conditions, see the discussion on p. 164-168 of [5].) The effect of the change of measure is to reduce the dimension of the underlying driving Markov process of (3) from three to two. That is, the driving source of randomness changes from $\left\{\left(t, X_{t}, \bar{X}_{t}\right): t \geqslant 0\right\}$ to $\left\{\left(t,\left(x \vee \bar{X}_{t}\right)-X_{t}\right): t \geqslant 0\right\}$. The Shepp-Shiryaev optimal stopping problem can be solved whenever it is possible to solve

$$
U(x)=\sup _{\tau \in \mathscr{T}_{0, \infty}} \mathbf{E}^{1}\left[e^{-\alpha \tau+Y_{\tau}^{x}} \mathbf{1}_{\{\tau<\infty\}}\right],
$$

where $\alpha=q-\psi(1)$ and $Y_{\tau}^{x}=\left(x \vee \bar{X}_{\tau}\right)-X_{\tau}$. The same effect occurs when the change of measure is applied to (1) in the case that $q>0$ and thus the 
expected pay-off function of the Shepp-Shiryaev game can be rewritten as

$$
M_{x}(\tau, \sigma)=\mathbf{E}^{1}\left[e^{-\alpha \tau+Y_{\tau}^{x}} \mathbf{1}_{\{\tau \leqslant \sigma, \tau<\infty\}}+e^{-\alpha \sigma}\left(e^{Y_{\sigma}^{x}}+\delta\right) \mathbf{1}_{\{\sigma<\tau\}}\right]
$$

when $q>0$. Establishing the saddle point $\left(\tau^{*}, \sigma^{*}\right)$ of $(1)$ or equivalently (6) in the case $q>0$ and of (2) in the case $q=0$, as well as establishing the value $V(x)=M\left(\tau^{*}, \sigma^{*}\right)$ in both cases is what is meant by solving Shepp-Shiryaev stochastic game. Note that a saddle point may not be unique. The purpose of this paper is to give a complete study of the solution of the Shepp-Shiryaev stochastic game within the specified parameter regime $q \geqslant 0$ and $\delta>0$.

In the Brownian motion case, the finite horizon version of (1) (i.e., when both players have to choose stopping times valued in $[0, T]$ for some $T>0$ ) was solved in the preprint [13] preceding [14] by decomposing it into two finite horizon optimal stopping problems, just as was done for the McKean stochastic game.

2. The solution to the Shepp-Shiryaev stochastic game. Below, in Theorems 2, 3, and 4 we give a qualitative and quantitative exposition of the solution to (1). Before doing so, we need to give a brief reminder of a class of special functions which appear commonly in connection with the study of spectrally negative Lévy processes and indeed in connection with the description of the Shepp-Shiryaev stochastic game as given below. For each $q \geqslant 0$ we introduce the functions $W^{(q)}: \mathbf{R} \rightarrow[0, \infty)$ which are known to satisfy for all $x \in \mathbf{R}$ and $a \geqslant 0$

$$
\mathbf{E}_{x}\left[e^{-q \tau_{a}^{+}} \mathbf{1}_{\left\{\tau_{a}^{+}<\tau_{0}^{-}\right\}}\right]=\frac{W^{(q)}(x \wedge a)}{W^{(q)}(a)}
$$

where $\tau_{a}^{+}:=\inf \left\{t>0: X_{t}>a\right\}$ and $\tau_{0}^{-}=\inf \left\{t>0: X_{t}<0\right\}$ (cf. [16, Chap. 8]). In particular, it is evident that $W^{(q)}(x)=0$ for all $x<0$ and further it is known that $W^{(q)}$ is almost everywhere differentiable on $(0, \infty)$, there is right-continuity at zero and

$$
\int_{0}^{\infty} e^{-\beta x} W^{(q)}(x) d x=\frac{1}{\psi(\beta)-q}
$$

for all $\beta>\Phi(q)$, where $\Phi(q)$ is the largest root of the equation $\psi(\theta)=q$ (of which there are at most two). For convenience, we write $W$ instead of $W^{(0)}$.

Associated to the functions $W^{(q)}$ are the functions $Z^{(q)}: \mathbf{R} \rightarrow[1, \infty)$ defined by

$$
Z^{(q)}(x)=1+q \int_{0}^{x} W^{(q)}(y) d y
$$

for $q \geqslant 0$. Together, the functions $W^{(q)}$ and $Z^{(q)}$ are collectively known as scale functions and predominantly appear in almost all fluctuation identities for spectrally negative Lévy processes. For example, it is also known that 
for all $x \in \mathbf{R}$ and $a, q \geqslant 0$,

$$
\mathbf{E}_{x}\left[e^{-q \tau_{0}^{-}} \mathbf{1}_{\left\{\tau_{a}^{+}>\tau_{0}^{-}\right\}}\right]=Z^{(q)}(x \wedge a)-\frac{Z^{(q)}(a)}{W^{(q)}(a)} W^{(q)}(x \wedge a) .
$$

We shall henceforth assume that

the jump measure of $X, \Pi_{X}$, has no atoms when $X$ has bounded variation.

Then it is known from existing literature (cf. [16]) that $W^{(q)} \in C^{1}(0, \infty)$ and hence $Z^{(q)} \in C^{2}(0, \infty)$. For computational convenience we shall proceed with the above assumption on $X$. Recall that $X$ has bounded variation if and only if it can be written in the form $X_{t}=\mathrm{d} t-S_{t}$ for $t \geqslant 0$, where $\left\{S_{t}: t \geqslant 0\right\}$ is a driftless subordinator with jump measure $\nu$ satisfying $\nu(x, \infty)=\Pi_{X}(-\infty,-x)$ (and then must necessarily satisfy $\left.\int_{(0, \infty)}(1 \wedge x) \nu(d x)<\infty\right)$ and $\mathrm{d}$ is a strictly positive constant which is referred to as the drift. In that case, it is also known that $W^{(q)}(0)=1 / \mathrm{d}$ and otherwise, when $X$ has unbounded variation, $W^{(q)}(0)=0$.

For comparison with the main contributions of this paper (Theorems 2, 3, and 4), we review the solution to the Shepp-Shiryaev optimal stopping problem (3), the essential part of which can be found in [2]. For convenience, we shall first introduce a subclass of spectrally negative Lévy processes. Denote by $\mathscr{G}$ the general class of spectrally negative Lévy processes and introduce the subclass

$$
\begin{aligned}
\mathscr{H}_{q}=\{X \in \mathscr{G}: & \int_{(-\infty, 0)}(1 \wedge|x|) \Pi_{X}(d x)=\infty \text { or } \sigma>0 \text { or } \\
& \left.\sigma=0, \int_{(-\infty, 0)}(1 \wedge|x|) \Pi_{X}(d x)<\infty, \text { and } q<\mathrm{d}\right\},
\end{aligned}
$$

where we recall the constant $d$ is the drift in the case of bounded variation (see the previous paragraph). Also needed is the following class of stopping times defined for all $y \geqslant 0$ by

$$
T_{y}^{+}=\inf \left\{t>0: Y_{t}^{x} \geqslant y\right\} \quad \text { and } T_{y}^{-}=\inf \left\{t>0: Y_{t}^{x} \leqslant y\right\} .
$$

Finally, let us introduce the continuous function

$$
f(x)=Z^{(q)}(x)-q W^{(q)}(x),
$$

which will play an important role in characterizing optimal thresholds. Owing to the fact that $W^{(q)}(x)=e^{\Phi(q) x} W_{\Phi(q)}(x)$, where $W_{\Phi(q)}(x)$ plays the role of $W(x)$ under $\mathbf{P}^{\Phi(q)}$, we can differentiate $f$ and easily deduce that, when $q>\psi(1) \vee 0$, the function $f$ is strictly decreasing to $-\infty$ and hence within this regime

$$
k^{*}:=\inf \{x \geqslant 0: f(x) \leqslant 0\} \in[0, \infty) .
$$


In particular, when $q>\psi(1) \vee 0, k^{*}=0$ if and only if $X \in \mathscr{G} \backslash \mathscr{H}_{q}$. This follows from the fact that $Z^{(q)}(0)=1$ and $W^{(q)}(0)=0$ unless $X$ has bounded variation in which case $W^{(q)}(0)=1 / \mathrm{d}$.

In the sequel, when $U$ is attained by a stopping time in $\mathscr{T}_{0, \infty}$, we shall denote it by $\tau^{*}$. That is, when it exists, $\tau^{*}$ satisfies

$$
U(x)=\mathbf{E}\left[e^{-q \tau^{*}+\left(x \vee \bar{X}_{\tau^{*}}\right)} 1_{\left\{\tau^{*}<\infty\right\}}\right] .
$$

Theorem 1. Let $q \geqslant 0$.

(i) When $q \leqslant \psi(1)$, we have $U(x)=\infty$ which is not attained by any $\tau \in \mathscr{T}_{0, \infty}$.

(ii) When $\psi(1)<q=0$, we have

$$
U(x)=e^{x}+(\Phi(0)-1)^{-1} e^{x(1-\Phi(0))},
$$

for $x \geqslant 0$, which is not attained by any $\tau \in \mathscr{T}_{0, \infty}$.

(iii) When $X \in \mathscr{G} \backslash \mathscr{H}_{q}$, we have for $x \geqslant 0$

$$
U(x)=e^{x} \text { and } \tau^{*}=0 .
$$

(iv) When $q>\psi(1) \vee 0$ and $X \in \mathscr{H}_{q}$, we have

$$
U(x)=e^{x} Z^{(q)}\left(k^{*}-x\right) \quad \text { and } \quad \tau^{*}=T_{k^{*}}^{+} .
$$

P r o o f. Cases (iii) and (iv) are contained in [2, Theorem 2]. Suppose $q \leqslant \psi(1)$. Since $\sup _{t \geqslant 0} Y_{t}^{x}=\sup _{t \geqslant 0}\left(\left(x \vee \bar{X}_{t}\right)-X_{t}\right)$ is $\mathbf{P}^{1}$-almost surely unbounded, the sequence of stopping times $\left\{T_{n}^{+}\right\}_{n \in \mathbf{N}}$ is $\mathbf{P}^{1}$-almost surely finite. Hence when $\alpha \leqslant 0$, we have $U(x) \geqslant \mathbf{E}^{1}\left[e^{-\alpha T_{n}^{+}+Y_{T_{n}^{+}}^{x}}\right] \geqslant e^{n}$, which implies (i).

Suppose $\psi(1)<q=0$. Then by monotonicity of $\bar{X}$

$$
\mathbf{E}\left[e^{x \vee \bar{X}_{\infty}}\right] \geqslant \sup _{\tau \in \mathscr{T}_{0, \infty}} \mathbf{E}\left[e^{x \vee \bar{X}_{\tau}} \mathbf{1}_{\{\tau<\infty\}}\right] \geqslant \sup _{t \geqslant 0} \mathbf{E}\left[e^{x \vee \bar{X}_{t}}\right]=\mathbf{E}\left[e^{x \vee \bar{X}_{\infty}}\right] .
$$

Since $\psi(1)<0$, it follows that $\psi^{\prime}(0+)<0$ and hence by a well-known result for spectrally negative processes, $\bar{X}_{\infty}$ is exponentially distributed with parameter $\Phi(0)=\sup \{\theta \geqslant 0: \psi(\theta)=0\}>1$. Thus we deduce (ii). Theorem 1 is proved.

Note that when $X \in \mathscr{G} \backslash \mathscr{H}_{q}$ it follows that

$$
q \geqslant \mathrm{~d}>0 \vee\left(\mathrm{d}+\int_{(-\infty, 0)}\left(e^{x}-1\right) \Pi_{X}(d x)\right)=0 \vee \psi(1)
$$

and hence the four cases in the above theorem constitute an exhaustive partition of the regime $q \geqslant 0$ for the optimal stopping problem (3).

Now, turning to the solution of the Shepp-Shiryaev stochastic game, it turns out that it is necessary to divide the regime $q \geqslant 0$ into several subcases. We present our main results accordingly. 
Theorem 2 (the case $q=0$ ). If $q=0$, then the solution to the SheppShiryaev stochastic game (2) is given as follows:

(i) when $\psi(1) \geqslant 0$, we have for any $\delta>0$ that a saddle point is given by $\dot{\sigma}^{*}=0$ and $\tau^{*}=\infty$ and hence $V(x)=e^{x}+\delta$;

(ii) when $\psi(1)<0$ and $(\Phi(0)-1) \delta>1$, we have that $\tau^{*}=\sigma^{*}=\infty$ and

$$
V(x)=e^{x}+(\Phi(0)-1)^{-1} e^{x(1-\Phi(0))}, \quad \text { for } \quad x \geqslant 0
$$

and

(iii) when $\psi(1)<0$ and $(\Phi(0)-1) \delta \leqslant 1$, we have $\tau^{*}=\infty, \sigma^{*}=T_{0}^{-}$,

$$
V(x)=e^{x}+\delta e^{x(1-\Phi(0))} .
$$

Theorem 3 (the case $0<q<\psi(1)$ ). Suppose $0<q<\psi(1)$. Let $f$ be defined as in (10).

The equation

$$
f(y)=1 \quad \text { for } \quad y>0
$$

has a unique solution (which we denote by $y^{*}$ ). The solution to the SheppShiryaev stochastic game (6) is given as follows.

(i) If $\delta>Z^{(q)}\left(y^{*}\right)-1$, then

$$
V(x)= \begin{cases}e^{x}+\delta & \text { when } x<a^{*} \\ e^{x} Z^{(q)}\left(b^{*}-x\right) & \text { when } x \geqslant a^{*}\end{cases}
$$

where $0<a^{*}<b^{*}<\infty$ satisfy

$$
\begin{gathered}
Z^{(q)}\left(b^{*}-a^{*}\right)=1+\delta e^{-a^{*}}, \\
b^{*}=a^{*}+y^{*} .
\end{gathered}
$$

$A$ saddle point is given by $\sigma^{*}=T_{a^{*}}^{-}$and $\tau^{*}=T_{b^{*}}^{+}$. Further, the function $V(x)$ is monotone increasing and $V(x)-e^{x}$ is monotone decreasing.

(ii) If $\delta \leqslant Z^{(q)}\left(y^{*}\right)-1$, then there exists a unique $z^{*} \in\left(0, y^{*}\right]$ which satisfies $Z^{(q)}\left(z^{*}\right)=1+\delta$ and then

$$
V(x)=e^{x} Z^{(q)}\left(z^{*}-x\right)
$$

and a saddle point is given by $\sigma^{*}=T_{0}^{-}$and $\tau^{*}=T_{z^{*}}^{+}$.

Theorem 4 (the case $q>0$ and $q \geqslant \psi(1)$ ). Let $q>0$. Recall that $z^{*}$ is the unique solution of $Z^{(q)}(z)=1+\delta$ which always exists uniquely, since $Z^{(q)}$ is a strictly increasing continuous function with $Z^{(q)}(0)=1$ and $Z^{(q)}(\infty)=\infty$. Also, recall that for $q>0$ and $X \in \mathscr{H}_{q}$, the equation $f(x)=0$ has a unique solution, denoted by $k^{*}$. The solution to the Shepp-Shiryaev stochastic game (6) is given as follows.

(i) When $q=\psi(1)$ and $\delta>0$, we have $\sigma^{*}=T_{0}^{-}, \tau^{*}=T_{z^{*}}^{+}$, and

$$
V(x)=e^{x} Z^{(q)}\left(z^{*}-x\right) .
$$


(ii) When $q>\psi(1), X \in \mathscr{H}_{q}$, and $\delta>Z^{(q)}\left(k^{*}\right)-1$ (so that $\left.k^{*}<z^{*}\right)$, we have $\sigma^{*}=\infty, \tau^{*}=T_{k^{*}}^{+}$and

$$
V(x)=U(x)=e^{x} Z^{(q)}\left(k^{*}-x\right) .
$$

(iii) When $q>\psi(1), X \in \mathscr{H}_{q}$, and $\delta \leqslant Z^{(q)}\left(k^{*}\right)-1$ (so that $k^{*} \geqslant z^{*}$ ), we have $\sigma^{*}=T_{0}^{-}, \tau^{*}=T_{z^{*}}^{+}$and

$$
V(x)=e^{x} Z^{(q)}\left(z^{*}-x\right) .
$$

(iv) When $q>\psi(1)$ and $X \in \mathscr{G} \backslash \mathscr{H}_{q}$, we have for any $\delta>0$ that $\tau^{*}=0$ and $\sigma^{*}=\infty$ form a saddle point, hence

$$
V(x)=e^{x} .
$$

$\mathrm{R}$ e $\mathrm{m}$ a $\mathrm{rk} 1$ (intuition). We briefly discuss some of the intuition behind the results of Theorems 2,3 , and 4 .

When $q=0$, one might expect it not to be optimal for the sup-player to stop, since the gain in (2) is nondecreasing in time. One would also expect the inf-player to never stop when the penalty $\delta$ is too large, which is indeed the conclusion of Theorem 2 (ii). When $\psi(1) \geqslant 0$, we have

$$
\mathbf{E}\left[e^{\bar{X}_{t} \vee x}\right] \geqslant \mathbf{E}\left[e^{X_{t}}\right]=e^{\psi(1) t}
$$

which indicates that the inf-player cannot gain by waiting and hence should stop immediately. When $\psi(1)<0$ and $\delta$ is below a critical value, it becomes worthwhile for the inf-player to stop. Since $\psi(1)<0$ implies that $\mathbf{E}\left[e^{X_{t}}\right]$ decreases in $t$, it might be lucrative for the inf-player not to stop immediately and it turns out that it is optimal for the inf-player to stop when the reflected process $Y$ reaches its minimum 0 . Note that this stopping time is infinite with positive probability.

When $q \geqslant \psi(1)$ and $q>0$, we observe the same phenomenon that the inf-player stops when $Y$ reaches 0 providing $\delta$ is below a critical value. This time, since $q>0$, the sup-player should stop as well in an almost surely finite stopping time and indeed this happens at the first time $Y$ exceeds a certain positive value (possibly by a jump).

When $0<q<\psi(1)$, the discount factor $\alpha$ in (6) is negative and therefore the inf-player should stop at an almost surely finite time. It also seems plausible that the inf-player should stop sooner than when $\psi(1) \leqslant q$, resulting in an optimal stopping set of the form $\left[0, a^{*}\right]$. However, this only happens when the penalty $\delta$ is large enough. It might seem counterintuitive that the inf-player is more eager to stop when the penalty is large, but this strategy could be explained by reasoning that the inf-player is tolerant to the negative discount factor $\alpha$ in (6) as long as $\delta$ is small enough. When $\delta$ becomes too large, the inf-player needs to take evasive action by stopping sooner. 
$\mathrm{R} \mathrm{e} \mathrm{m}$ a r k 2 (pasting conditions). Theorems 3 and 4 both state that the value function of the Shepp-Shiryaev stochastic game necessarily takes the form

$$
V(x)= \begin{cases}\delta+e^{x} & \text { when } x<a, \\ e^{x} Z^{(q)}(b-x) & \text { when } x \geqslant a,\end{cases}
$$

for some $0 \leqslant a \leqslant b<\infty$. As a consequence of the behavior at the origin of the scale functions $Z^{(q)}$ and $W^{(q)}$, if follows that when $a>0$, there is smooth pasting at $a$ (in accordance with the fact that 0 is regular for $(0, \infty)$ for $X$ ). Further, when $b<\infty$ there always is continuity at $b$ and smooth pasting at $b$ if and only if $X$ has unbounded variation (corresponding to the case that 0 is regular for $(-\infty, 0)$ for $X)$. See [1] for a discussion on the relevance of path regularity to pasting conditions.

The rest of this paper is structured as follows. In the next section we make note of a Verification Lemma for the optimal stopping game (6) under the change of measure. This lemma essentially allows us to «verify» directly that the solutions presented in Theorems 3 and 4 are indeed optimal. In addition, we present the candidate functions which will be used in conjunction with the Verification Lemma to establish the solution. In Section 4 we give the proof of Theorem 3. Having done this, one sees that the proof of Theorem 4 is a straightforward variant of a part of the proof of Theorem 3. We only comment briefly in Section 5 on the proof of Theorem 4, which is otherwise left as an exercise for the reader. In Section 6 we give the proof of Theorem 2. The proof differs from the proofs of Theorems 3 and 4 in the sense that one may no longer appeal to the change of measure (4).

3. Preliminary results. Following classical ideas in optimal stopping, we verify that a candidate solution solves the Shepp-Shiryaev game by checking certain associated bounds and martingale properties. Specifically, we use the following verification lemma for the case $q>0$, which is a variant of the similar one in [3].

Lemma 1 (Verification Lemma). Suppose that $\tau^{*} \in \mathscr{T}_{0, \infty}$ and $\sigma^{*} \in \mathscr{T}_{0, \infty}$ are candidate optimal strategies for the stochastic game (6) such that

$$
e^{Y_{\sigma}^{x}} 1_{\left\{\sigma<\tau^{*}\right\}}
$$

is uniformly bounded by a constant for all $\sigma \in \mathscr{T}_{0, \infty}$ and $x \geqslant 0$. Let

$$
V^{*}(x)=\mathbf{E}^{1}\left[e^{-\alpha \tau^{*}+Y_{\tau^{*}}^{x}} \mathbf{1}_{\left\{\tau^{*} \leqslant \sigma^{*}, \tau^{*}<\infty\right\}}+e^{-\alpha \sigma^{*}}\left(e^{Y_{\sigma^{*}}^{x}}+\delta\right) \mathbf{1}_{\left\{\sigma^{*}<\tau^{*}\right\}}\right] .
$$

Then the pair $\left(\tau^{*}, \sigma^{*}\right)$ is a saddle point to (6) if

(i) $V^{*}(x) \geqslant e^{x}$,

(ii) $V^{*}(x) \leqslant e^{x}+\delta$,

(iii) $V^{*}\left(Y_{\tau^{*}}\right)=e^{Y_{\tau^{*}}}$ almost surely on $\left\{\tau^{*}<\infty\right\}$,

(iv) $V^{*}\left(Y_{\sigma^{*}}\right)=e^{Y_{\sigma^{*}}}+\delta$ almost surely on $\left\{\sigma^{*}<\infty\right\}$, 
(v) the process $\left\{e^{-\alpha\left(t \wedge \tau^{*}\right)} V^{*}\left(Y_{t \wedge \tau^{*}}\right)\right\}_{t \geqslant 0}$ is a right-continuous submartingale under $\mathbf{P}^{1}$, and

(vi) the process $\left\{e^{-\alpha\left(t \wedge \sigma^{*}\right)} V^{*}\left(Y_{t \wedge \sigma^{*}}\right)\right\}_{t \geqslant 0}$ is a right-continuous supermartingale under $\mathbf{P}^{1}$.

P r o of. Define for each $\tau, \sigma \in \mathscr{T}_{0, \infty}$

$$
\Theta_{\tau, \sigma}^{\alpha}=e^{-\alpha \tau+Y_{\tau}^{x}} 1_{\{\tau \leqslant \sigma, \tau<\infty\}}+e^{-\alpha \sigma}\left(e^{Y_{\sigma}^{x}}+\delta\right) 1_{\{\sigma<\tau\}} .
$$

From the supermartingale property (vi), Doob's optional stopping theorem, (iv), and (i) we know that for any $\tau \in \mathscr{T}_{0, \infty}$ and $t \geqslant 0$,

$$
\begin{aligned}
V^{*}(x) & \geqslant \mathbf{E}^{1}\left[e^{-\alpha\left(t \wedge \tau \wedge \sigma^{*}\right)} V^{*}\left(X_{t \wedge \tau \wedge \sigma^{*}}\right)\right] \\
& \geqslant \mathbf{E}^{1}\left[e^{-\alpha(t \wedge \tau)+Y_{t \wedge \tau}^{x}} \mathbf{1}_{\left\{\sigma^{*} \geqslant t \wedge \tau, \tau<\infty\right\}}+e^{-\alpha \sigma^{*}}\left(e^{Y_{\sigma^{*}}^{x}}+\delta\right) \mathbf{1}_{\left\{\sigma^{*}<t \wedge \tau\right\}}\right] .
\end{aligned}
$$

By taking limits $t \rightarrow \infty$, it follows from Fatou's lemma that

$$
V^{*}(x) \geqslant \mathbf{E}^{1}\left[\Theta_{\tau, \sigma^{*}}^{\alpha}\right] .
$$

Next, we show that

$$
V^{*}(x) \leqslant \mathbf{E}^{1}\left[\Theta_{\tau^{*}, \sigma}^{\alpha}\right] .
$$

If $\sigma$ is such that $\mathbf{E}^{1}\left[e^{-\alpha \sigma} \mathbf{1}_{\left\{\sigma<\tau^{*}\right\}}\right]=\infty$, then (16) holds trivially, since $V^{*}(x) \leqslant \infty$. Hence, we assume that $\sigma \in \mathscr{T}_{0, \infty}$ satisfies $\mathbf{E}^{1}\left[e^{-\alpha \sigma} \mathbf{1}_{\left\{\sigma<\tau^{*}\right\}}\right]<\infty$. Using (v), Doob's optional stopping theorem, (ii), and (iii) we find

$$
\begin{aligned}
V^{*}(x) & \leqslant \mathbf{E}^{1}\left[e^{-\alpha\left(t \wedge \tau^{*} \wedge \sigma\right)} V^{*}\left(X_{t \wedge \tau^{*} \wedge \sigma}\right)\right] \\
& =\mathbf{E}^{1}\left[e^{-\alpha \tau^{*}} V^{*}\left(X_{\tau^{*}}\right) \mathbf{1}_{\left\{\tau^{*} \leqslant t \wedge \sigma\right\}}+e^{-\alpha(t \wedge \sigma)} V^{*}\left(X_{t \wedge \sigma}\right) \mathbf{1}_{\left\{\tau^{*}>t \wedge \sigma\right\}}\right] \\
& \leqslant \mathbf{E}^{1}\left[e^{-\alpha \tau^{*}+Y_{\tau^{*}}^{x}} \mathbf{1}_{\left\{\tau^{*} \leqslant t \wedge \sigma\right\}}+e^{-\alpha(t \wedge \sigma)}\left(e^{Y_{t \wedge \sigma}^{x}}+\delta\right) \mathbf{1}_{\left\{\tau^{*}>t \wedge \sigma\right\}}\right] .
\end{aligned}
$$

Taking limits as $t \uparrow \infty$ and applying the monotone convergence theorem to the first term on the right-hand side and the dominated convergence theorem (see (14)) to the second term on the right-hand side, we find that indeed (16) is fulfilled and hence the saddle point is achieved with the strategies $\left(\tau^{*}, \sigma^{*}\right)$. Lemma 1 is proved.

Note that Lemma 1 implies that when $\delta \geqslant \sup _{x \geqslant 0}\left(U(x)-e^{x}\right)$, a solution to the game is given by taking $V^{*}=U$ and $\tau^{*}$ as in Theorem 1 and $\sigma^{*}=\infty$. This agrees with the intuition that the inf-player will force a greater payment by stopping than the sup-player would otherwise induce by stopping and hence it is better for the inf-player not to stop at all.

We shall often apply the above verification lemma to solutions of the form $V(x, a, b)$ for $0 \leqslant a<x<b<\infty$ corresponding to taking strategies $\sigma=T_{a}^{-}$and $\tau=T_{b}^{+}$in (1). That is,

$$
V(x, a, b)=\mathbf{E}^{1}\left[e^{-\alpha T_{b}^{+}+Y_{T_{b}^{+}}^{x}} \mathbf{1}_{\left\{T_{b}^{+} \leqslant T_{a}^{-}\right\}}+e^{-\alpha T_{a}^{-}}\left(e^{Y_{T_{a}^{-}}^{x}}+\delta\right) \mathbf{1}_{\left\{T_{a}^{-}<T_{b}^{+}\right\}}\right] .
$$

Using fluctuation theory we prove the following result. 
Lemma 2. Let $0 \leqslant a<x<b<\infty$. Then

$$
\begin{gathered}
V(x, a, b)=e^{x}\left(Z^{(q)}(b-x)-W^{(q)}(b-x) \frac{Z^{(q)}(b-a)}{W^{(q)}(b-a)}\right. \\
\left.+\left(1+\delta e^{-a}\right) \frac{W^{(q)}(b-x)}{W^{(q)}(b-a)}\right) .
\end{gathered}
$$

$\mathrm{Pr}$ o of. Let $0 \leqslant a<x<b<\infty$. Note that $\tau_{x-b}^{-}=T_{b}^{+}$on the event $\left\{T_{b}^{+}<T_{a}^{-}\right\}$and $\tau_{x-a}^{+}=T_{a}^{-}$on the event $\left\{T_{a}^{-}<T_{b}^{+}\right\}$. We change measure using (4), then use (7) and (9) to derive

$$
\begin{aligned}
V(x, a, b)= & \mathbf{E}^{1}\left[e^{-\alpha T_{b}^{+}+Y_{T_{b}^{+}}^{x}} \mathbf{1}_{\left\{T_{b}^{+} \leqslant T_{a}^{-}\right\}}+e^{-\alpha T_{a}^{-}}\left(e^{Y_{T_{a}^{-}}^{x}}+\delta\right) \mathbf{1}_{\left\{T_{a}^{-}<T_{b}^{+}\right\}}\right] \\
= & \mathbf{E}\left[e^{-q \tau_{x-b}^{-}+\left(x \sqrt{X_{\tau_{x-b}^{-}}}\right)} \mathbf{1}_{\left\{\tau_{x-b}^{-} \leqslant \tau_{x-a}^{+}\right\}}\right] \\
& +\left(\delta+e^{a}\right) \mathbf{E}\left[e^{-q \tau_{x-a}^{+}+X_{\tau_{x-a}^{+}}} \mathbf{1}_{\left\{\tau_{x-a}^{+}<\tau_{b-x}^{-}\right\}}\right]
\end{aligned}
$$

from which (18) follows. Lemma 2 is proved.

4. Proof of Theorem 3. We begin with a preliminary lemma (from which the opening part of Theorem 3 follows) concerning the function $f(x)$ defined in (10).

Lemma 3. Suppose $0<q<\psi(1)$. The equation $f(y)=1$ has a unique solution (denoted by $\left.y^{*}\right)$ on $(0, \infty)$ such that $0<f(x)<1$ for $0<x<y^{*}$ and $f^{\prime}\left(y^{*}\right)>0$.

P r o of. In the case when $X$ has unbounded variation, we have $f(0)=$ 1 and

$$
f^{\prime}(0+)=-q W^{(q) \prime}(0+)=-q \lim _{\lambda \rightarrow \infty} \frac{\lambda^{2}}{\psi(\lambda)-q}= \begin{cases}-2 q \sigma^{-2} & \text { when } \quad \sigma>0, \\ -\infty & \text { when } \quad \sigma=0 .\end{cases}
$$

In the case of bounded variation with drift $\mathrm{d}$, we have $f(0)=1-q / \mathrm{d}<1$. Also $\mathrm{d}>\psi(1)>q$, so $f(0)>0$. Hence in either case of bounded or unbounded variation, it follows that $f(\varepsilon)<1$ for some $\varepsilon>0$.

Recalling that $W^{(q)}(x)=e^{\Phi(q) x} W_{\Phi(q)}(x)$, we have for $x>0$

$$
\begin{aligned}
f^{\prime}(x) & =q\left(W^{(q)}(x)-W^{(q) \prime}(x)\right) \\
& =q e^{\Phi(q) x}\left([1-\Phi(q)] W_{\Phi(q)}(x)-W_{\Phi(q)}^{\prime}(x)\right) .
\end{aligned}
$$

It is also known that $W_{\Phi(q)}^{\prime}(x)=W_{\Phi(q)}(x) n_{\Phi(q)}(h>x)$, where $n_{\Phi(q)}$ is the excursion measure of $\bar{X}-X$ under $\mathbf{P}^{\Phi(q)}$. Hence,

$$
f^{\prime}(x)=q W^{(q)}(x)\left(1-\Phi(q)-n_{\Phi(q)}(h>x)\right)
$$

and thus, in particular, $f(\infty)=\infty$ implying that the function $f$ attains its minimum. From (19) it also follows that $f^{\prime}(x) \geqslant 0$ for some $x$ implies that 
$f^{\prime}(y)>0$ for all $y>x$. From the first paragraph of this proof we deduce that the minimum of $f$ is valued in $(-\infty, 1)$ and that this minimum is uniquely attained (say at $m$ ). We deduce that the equation $f(y)=1$ has a unique solution on $(0, \infty)$ (denoted by $\left.y^{*}\right)$. Clearly, $y^{*}>m$ and it readily follows that $f^{\prime}\left(y^{*}\right)>0$ and that $f(x)<1$ for all $0<x<y^{*}$.

We now show positivity of $f$. It is known from the Wiener-Hopf factorization (cf. [16, Chap. 8]) that

$$
\frac{1}{q}>\frac{1}{q} \mathbf{P}\left\{-\underline{X}_{\mathbf{e}_{\mathbf{q}}} \in[0, x]\right\}=\frac{1}{\Phi(q)} W^{(q)}(x)-\int_{0}^{x} W^{(q)}(y) d y,
$$

where $\mathbf{e}_{\mathbf{q}}$ is an independent and exponentially distributed random variable with parameter $q$ and $\underline{X}_{t}=\inf _{s \leqslant t} X_{s}$. Since $0<q<\psi(1)$, it follows that $-\Phi(q)^{-1}<-1$ and hence

$f(x)>Z^{(q)}(x)-\frac{q}{\Phi(q)} W^{(q)}(x)=1-q\left(\frac{1}{\Phi(q)} W^{(q)}(x)-\int_{0}^{x} W^{(q)}(y) d y\right)>0$,

which completes the proof.

We now divide the forthcoming analysis into the two cases $\delta>$ $Z^{(q)}\left(y^{*}\right)-1$ and $\delta \leqslant Z^{(q)}\left(y^{*}\right)-1$ corresponding to parts (i) and (ii), respectively, of Theorem 3 .

4.1. The case $\delta>Z^{(q)}\left(y^{*}\right)-1$. Under this subregime of $0<q<\psi(1)$, we have the existence of $0<a^{*}<b^{*}<\infty$ satisfying

$$
Z^{(q)}\left(y^{*}\right)=1+\delta e^{-a^{*}} \text { and } b^{*}=a^{*}+y^{*}
$$

where $y^{*}$ was defined as the unique solution in $(0, \infty)$ of $(11)$. Note that this choice of $a^{*}$ and $b^{*}$ has the convenient implication that for $x \geqslant a^{*}$

$$
V\left(x, a^{*}, b^{*}\right)=e^{x} Z^{(q)}\left(b^{*}-x\right) .
$$

From the latter, we see that $V\left(x, a^{*}, b^{*}\right)>e^{x}$ on $\left[a^{*}, b^{*}\right)$. Moreover, $V^{\prime}\left(x, a^{*}, b^{*}\right)=e^{x} f\left(b^{*}-x\right)$ and, on account of the fact that $f\left(b^{*}-x\right)<1$ for all $x>a^{*}$, it follows that

$$
V\left(x, a^{*}, b^{*}\right)<e^{x}+\delta \quad \text { for all } x>a^{*} .
$$

Since $f$ is positive, it also follows that $V\left(x, a^{*}, b^{*}\right)$ is increasing in $x$, and thus, in particular,

$$
e^{a^{*}}+\delta=V\left(a^{*}, a^{*}, b^{*}\right)<V\left(b^{*}, a^{*}, b^{*}\right)=e^{b^{*}} .
$$

Next, define the function $\theta: \mathbf{R} \rightarrow \mathbf{R}$ by

$$
\theta(x)=Z^{(q)}\left(b^{*}-x\right)-1-\delta e^{-x} .
$$

We will shortly make use of the following lemma.

Lemma 4. The function $\theta$ satisfies $\theta\left(a^{*}\right)=0$ and $\theta(x)<0$ for all $x<a^{*}$. 
P r o o f. The statement $\theta\left(a^{*}\right)=0$ rephrases (12). Next, differentiating and using the fact that $b^{*}-a^{*}=y^{*}$ (and hence $f\left(b^{*}-a^{*}\right)=1$ ), we have

$$
\theta^{\prime}\left(a^{*}\right)=-q W^{(q)}\left(b^{*}-a^{*}\right)+\delta e^{-a^{*}}=1-Z^{(q)}\left(b^{*}-a^{*}\right)+\delta e^{-a^{*}}=0 .
$$

From Lemma 3 we have $f^{\prime}\left(y^{*}\right)>0$ and hence $W^{(q) \prime}\left(b^{*}-a^{*}\right)<W^{(q)}\left(b^{*}-a^{*}\right)$, which in turn implies that

$$
\theta^{\prime \prime}\left(a^{*}\right)=q W^{(q) \prime}\left(b^{*}-a^{*}\right)-\delta e^{-a^{*}}<q W^{(q)}\left(b^{*}-a^{*}\right)-\delta e^{-a^{*}}=0 .
$$

In particular, $\theta(x)<0$ for all $x \in\left(a^{*}-\varepsilon, a^{*}\right)$ and some sufficiently small $\varepsilon>0$.

Suppose now, for contradiction, that $c=\sup \left\{x<a^{*}-\varepsilon: \theta(x)=0\right\}>$ $-\infty$. Then by Rolle's theorem, there exists $d \in\left(c, a^{*}\right)$ such that $\theta(d)<0$ and $\theta^{\prime}(d)=0$. On the other hand, for $x<a^{*}$ we also have

$$
\theta(x)+\theta^{\prime}(x)=f\left(b^{*}-x\right)-1>f\left(b^{*}-a^{*}\right)-1=0,
$$

where we have used Lemma 3. In particular with $x=d$ we find $\theta(d)>0$ which is in contradiction with the definition of $d$. In conclusion, $c=a^{*}$ and $\theta(x)<0$ for all $x<a^{*}$ as required. Lemma 4 is proved.

Our strategy for proving part (i) of Theorem 3 will be to look at an auxiliary optimal stopping problem and then use the above information to associate the solution of the aforementioned optimal stopping problem with the solution of the Shepp-Shiryaev stochastic game. To this end, let

$$
I(x)=\inf _{\sigma \in \mathscr{T}_{0, \infty}} \mathbf{E}^{1}\left[e^{-\alpha \sigma} g\left(\widetilde{Y}_{\sigma}^{x}\right)\right],
$$

where $\tilde{Y}_{\sigma}^{x}:=Y_{\sigma \wedge T_{b^{*}}}^{x}, g$ is any continuous function such that $g(x)=e^{x}+\delta$ when $x<a^{*}$ and $g(x)=e^{x}$ when $x \geqslant b^{*}$ and $e^{x}+\delta>g(x)>e^{x} Z^{(q)}\left(b^{*}-x\right)$ for any $x \in\left(a^{*}, b^{*}\right)$.

Theorem 5. There exists a solution to the optimal stopping problem (25) with the following properties.

(i) For $x>a^{*}, I(x)=V\left(x, a^{*}, b^{*}\right)$.

(ii) For all $x \in\left(0, b^{*}\right), I(x)>e^{x}$.

$\mathrm{P} \mathrm{r}$ o o f. By taking $\sigma=0$ in the expectation on the right-hand side of (25), we see that $I(x) \leqslant e^{x}+\delta$. Hence, it follows that $I(x)=I(x) \wedge$ $\left(e^{x}+\delta\right)=\inf _{\sigma \in \mathscr{O}_{0, \infty}} \mathbf{E}^{1}\left[\left(e^{-\alpha \sigma} g\left(\tilde{Y}_{\sigma}^{x}\right)\right) \wedge\left(e^{x}+\delta\right)\right]$ and (25) is an optimal stopping problem for a strong Markov process, where, for each fixed $x \geqslant 0$, the pay-off function is continuous and bounded but as a function of $x$ the stochastic gain is locally bounded in $x$.

Taking note of (2.2.80) in [21], we may now invoke Corollary 2.9 in the same reference to deduce the existence of an optimal stopping time $\sigma^{*}$ in (25) which is of the form $\sigma^{*}=\inf \left\{t>0: \widetilde{Y}_{t}^{x} \in D\right\}$, where $D=\{x: I(x)=g(x)\}$. 
Since $\alpha=q-\psi(1)<0$ and $g(x) \geqslant 1+\delta$, we have that $0 \in D$. Now, define

$$
s:=\sup \left\{0 \leqslant x<b^{*}: I(x)=g(x)\right\} .
$$

Taking $\sigma=T_{a^{*}}^{-}$in the expectation on the right-hand side of (25) leads to a value of $V\left(x, a^{*}, b^{*}\right)$ and thus for any $x>a^{*}$ it holds that $I(x) \leqslant$ $V\left(x, a^{*}, b^{*}\right)<e^{x}+\delta$, where the last inequality follows by virtue of (21). As a consequence, we now see that $s \leqslant a^{*}$.

(i) We want to rule out the case that $s<a^{*}$ and then part (i) will follow. Suppose for contradiction that $s<a^{*}$. Then, on $\left[s, b^{*}\right]$ we have $I=V\left(\cdot, s, b^{*}\right)$. In particular, it holds that

$$
\begin{aligned}
I^{\prime}(s+)=V^{\prime}\left(s, s, b^{*}\right)=e^{s}+\delta+e^{s}( & -q W^{(q)}(b-s)+\frac{W^{(q) \prime}(b-s)}{W^{(q)}(b-s)} \\
& \left.\times\left(Z^{(q)}(b-s)-1-\delta e^{-s}\right)\right) .
\end{aligned}
$$

The fact that $0<f^{\prime}\left(b^{*}-a^{*}\right)$ implies that $W^{(q) \prime}\left(b^{*}-s\right) / W^{(q)}\left(b^{*}-s\right)<1$ and thus, using Lemma 4 we find

$$
I^{\prime}(s)>e^{s}+\delta+e^{s}\left(-q W^{(q)}\left(b^{*}-s\right)+Z^{(q)}\left(b^{*}-s\right)-1-\delta e^{-s}\right)>e^{s},
$$

where the last inequality follows from the fact that $f\left(b^{*}-s\right)>f\left(b^{*}-a^{*}\right)=1$. Since $I(s)=e^{s}+\delta$, the previous calculations indicate that $I$ violates its upper bound $e^{x}+\delta$. We conclude that $s=a^{*}$ and thus $\left(a^{*}, b^{*}\right) \subseteq D^{c}$.

(ii) The next step in the proof is to show that for all $x \in\left[0, b^{*}\right)$

$$
I(x)>e^{x} .
$$

We prove (26) by contradiction. First, we show that there are only a finite number of intervals $(l, r)$ satisfying $(l, r) \subset D^{c}, 0 \leqslant l<r \leqslant a^{*}$, $I(l)=e^{l}+\delta, I(r)=e^{r}+\delta$ and such that there is some $x \in(l, r)$ for which $I(x) \leqslant e^{x}$. Indeed, since $\alpha<0$, taking into account the fact that optimal stopping occurs whenever $\tilde{Y}^{x}$ hits the domain $D$ and that $X$ is spectrally negative, we deduce that for any $x \in(l, r)$, with $(l, r)$ an interval satisfying the properties above,

$$
I(x) \geqslant e^{l}+\delta
$$

since $\left(a^{*}, b^{*}\right) \subset D^{c}$, and since from (22) it follows that $\inf _{x \in D \cap[r, \infty)} g(x)=$ $e^{r}+\delta>e^{l}+\delta$. Hence, whenever $x \in(l, r)$ satisfies $I(x)<e^{x}$, then it must hold that $x \geqslant \ln \left(e^{l}+\delta\right)$. In particular, $(l, r)$ is necessarily of minimal length $\ln \left(e^{l}+\delta\right)-l$ and therefore there can be only finitely many intervals of this form. Now let $\left(l^{*}, r^{*}\right)$ be the rightmost of such intervals. Fix $x \in\left(l^{*}, r^{*}\right)$ and set

$$
T_{\left(l^{*}, r^{*}\right)}=\inf \left\{t>0: Y_{t}^{x} \notin\left(l^{*}, r^{*}\right)\right\} .
$$


Note that $T_{\left(l^{*}, r^{*}\right)} \leqslant T_{D}$, where

$$
T_{D}=\inf \left\{t>0: Y_{t}^{x} \in D\right\} .
$$

Since $\left\{e^{-\alpha\left(t \wedge T_{D}\right)} I\left(Y_{t \wedge T_{D}}^{x}\right)\right\}$ is a $\mathbf{P}^{1}$-martingale (cf. [21, Theorem 2.4]) we have

$$
\begin{aligned}
I(x) & =\mathbf{E}^{1}\left[e^{-\alpha T_{\left(l^{*}, r^{*}\right)}} I\left(Y_{T_{\left(l^{*}, r^{*}\right)}}^{x}\right)\right] \\
& =\mathbf{E}^{1}\left[e^{-\alpha T_{\left(l^{*}, r^{*}\right)}}\left(\left(e^{Y_{l^{*}}^{x}}+\delta\right) \mathbf{1}_{\left\{T_{l^{*}}<T_{r^{*}}^{+}\right\}}+I\left(Y_{T_{r^{*}}^{+}}^{x}\right) \mathbf{1}_{\left\{T_{r^{*}}^{+}<T_{l^{*}}^{-}\right\}}\right)\right] \\
& \geqslant \mathbf{E}^{1}\left[e^{-\alpha T_{\left(l^{*}, r^{*}\right)}}\left(\left(e^{Y_{l^{*}}^{x}}+\delta\right) \mathbf{1}_{\left\{T_{l^{*}}<T_{r^{*}}^{+}\right\}}+e^{Y_{r_{r^{*}}^{+}}^{x}} \mathbf{1}_{\left\{T_{r^{*}}^{+}<T_{l^{*}}^{-}\right\}}\right)\right] \\
& =V\left(x, l^{*}, r^{*}\right),
\end{aligned}
$$

where for the inequality we used the fact that we have chosen $\left(l^{*}, r^{*}\right)$ as the rightmost interval on which $I(x)>e^{x}$ fails. Since $r^{*} \leqslant b^{*}$, we have for $x \in\left(l^{*}, r^{*}\right)$

$$
\begin{aligned}
V\left(x, l^{*}, r^{*}\right)= & e^{x}\left(Z^{(q)}\left(r^{*}-x\right)-W^{(q)}\left(r^{*}-x\right) \frac{Z^{(q)}\left(r^{*}-l^{*}\right)}{W^{(q)}\left(r^{*}-l^{*}\right)}\right. \\
& \left.\quad+\left(1+\delta e^{-l^{*}}\right) \frac{W^{(q)}\left(r^{*}-x\right)}{W^{(q)}\left(r^{*}-l^{*}\right)}\right) \\
\geqslant & e^{x}\left(Z^{(q)}\left(r^{*}-x\right)-\frac{W^{(q)}\left(r^{*}-x\right)}{W^{(q)}\left(r^{*}-l^{*}\right)}\left(Z^{(q)}\left(b^{*}-l^{*}\right)-1-\delta e^{-l^{*}}\right)\right) \\
\geqslant & e^{x} Z^{(q)}\left(r^{*}-x\right)>e^{x},
\end{aligned}
$$

where we have used Lemma 4 . This contradiction has the desired implication that $I(x)>e^{x}$ for all $x<a^{*}$. Theorem 5 is proved.

In the next result we establish that there exists a saddle point for the Shepp-Shiryaev stochastic game. Recall that $I(x)$ is specified by (25), where the function $g$ is arbitrary up to the specified constraints following (25).

Proposition 1. Let $0<q<\psi(1)$ and $\delta>Z^{(q)}\left(y^{*}\right)-1$. The stochastic game (1) has a solution and its value satisfies $V(x)=I(x)$ for all $x \geqslant 0$.

P r o o f. The proof uses the Verification Lemma 1 applied to the function $V^{*}=I$ and the stopping times $\tau^{*}=T_{b^{*}}^{+}$and

$$
\sigma^{*}=\inf \left\{t>0: Y_{t}^{x} \in D \cap\left[0, a^{*}\right]\right\},
$$

where $D=\{x \geqslant 0: I(x)=g(x)\}$.

From Theorem 5 we have that $I(x)$ fulfils conditions (i)-(iv) of Lemma 1. By standard optimal stopping theory and the strong Markov property, the submartingale property (v) automatically holds, see, for example, Theorem 2.4 of [21]. It remains to justify the remaining condition (vi). By

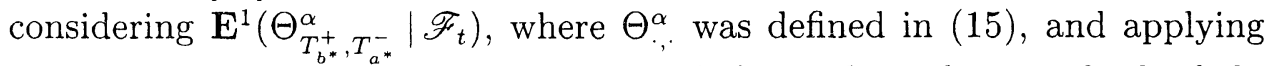
the strong Markov property, it is straightforward to show with the help of (20) that $\left\{e^{-\alpha t} e^{Y_{t}^{x}} Z^{(q)}\left(b^{*}-Y_{t}^{x}\right): t<T_{b^{*}}^{+} \wedge T_{a^{*}}^{-}\right\}$is a $\mathbf{P}^{1}$-martingale. Since 
$I(x)=e^{x} Z^{(q)}\left(b^{*}-x\right)$ for $x \geqslant a^{*}$ it holds that $I$ belongs to $C^{2}\left(a^{*}, b^{*}\right)$ and one may apply Itô's formula for semimartingales (cf. [22, Theorem 32 on p. 78]) to deduce that $(\Gamma-\alpha) I(x)=0$ on $\left(a^{*}, b^{*}\right)$, where $\Gamma$ is the infinitesimal generator of $-X$ under $\mathbf{P}^{1}$. Note also that since $\left\{e^{-X_{t}-\psi(1) t}: t \geqslant 0\right\}$ is a martingale under $\mathbf{P}^{1}$ we have that $(\Gamma-\alpha) e^{x}=-q e^{x}$ for all $x \in \mathbf{R}$. It now follows that $(\Gamma-\alpha) I(x) \leqslant 0$ for $x>b^{*}$. Appealing to the known behavior of the scale function $Z^{(q)}$ at zero (cf. [16, Chap. 8]), if $X$ has bounded variation then $I$ belongs to $C^{2}\left[\left(a^{*}, b^{*}\right) \cup\left(b^{*}, \infty\right)\right] \cap C^{0}\left(a^{*}, \infty\right)$ and otherwise, when $X$ has unbounded variation, $I$ belongs to $C^{2}\left[\left(a^{*}, b^{*}\right) \cup\left(b^{*}, \infty\right)\right] \cap C^{1}\left(a^{*}, \infty\right)$. In the first case, the version of the change of variable formula (which is nothing more than a special case of Itô's formula for semimartingales) in [17] may be used, and in the second case the version of Itô's formula established in [19] or [7] may be used together with the fact that $(\Gamma-\alpha) I(x) \leqslant 0$ for $x \in\left(a^{*}, b^{*}\right) \cup\left(b^{*}, \infty\right)$ to deduce that $\left\{e^{-\alpha t} e^{Y_{t}^{x}} Z^{(q)}\left(b^{*}-Y_{t}^{x}\right): t<T_{a^{*}}^{-}\right\}$is a supermartingale.

Note that right-continuity of the paths of all the above-mentioned semimartingales is clear on account of the continuity of $I$ and the right-continuity of $Y^{x}$. Proposition 1 is proved.

Were it not for the fact that we have not yet proved that $I(x)=e^{x}+\delta$ for all $x \leqslant a^{*}$, i.e., that $D \cap\left[0, a^{*}\right]=\left[0, a^{*}\right]$, we would be able to claim that the proof of Theorem 3 (i) is now complete. However, we must still rule out the possibility that $I(x)<e^{x}+\delta$ for some interval $\varnothing \neq(l, r) \subseteq\left[0, a^{*}\right]$. We finish this subsection by excluding this possibility and hence concluding the proof of Theorem 3 (i).

Theorem 6. The value function $I(x)-e^{x}$ is decreasing and hence part (i) of Theorem 3 holds.

P r o of. Let $x>y \geqslant 0$. We use the notation $\sigma(x)$ to make explicit the dependency of the stopping time $\sigma \in \mathscr{T}_{0, \infty}$ on the initial position of the process $Y^{x}$. We then find that for any $x \geqslant 0$

$$
\begin{aligned}
V(x) \leqslant & \mathbf{E}\left[e^{-q \tau^{*}(x)+\left(x \vee \bar{X}_{\tau^{*}(x)}\right)} \mathbf{1}_{\left\{\tau^{*}(x) \leqslant \sigma^{*}(y)\right\}}\right] \\
& +\mathbf{E}\left[e^{-q \sigma^{*}(y)}\left(e^{x \vee \bar{X}_{\sigma^{*}(y)}}+\delta e^{X_{\sigma^{*}}(y)}\right) \mathbf{1}_{\left\{\sigma^{*}(y)<\tau^{*}(x)\right\}}\right]
\end{aligned}
$$

and similarly, for any $y \geqslant 0$

$$
\begin{aligned}
V(y) \geqslant & \mathbf{E}\left[e^{-q \tau^{*}(x)+\left(y \vee \bar{X}_{\tau^{*}(x)}\right)} \mathbf{1}_{\left\{\tau^{*}(x) \leqslant \sigma^{*}(y)\right\}}\right] \\
& +\mathbf{E}\left[e^{-q \sigma^{*}(y)}\left(e^{y \vee \bar{X}_{\sigma^{*}(y)}}+\delta e^{X_{\sigma^{*}(y)}}\right) \mathbf{1}_{\left\{\sigma^{*}(y)<\tau^{*}(x)\right\}}\right] .
\end{aligned}
$$

Now, let $x>y \geqslant 0$. Then

$$
\begin{aligned}
V(x)-V(y) \leqslant & \mathbf{E}\left[e^{-q \tau^{*}(x)}\left(e^{x \vee \bar{X}_{\tau^{*}(x)}}-e^{y \vee \bar{X}_{\tau^{*}(x)}}\right) \mathbf{1}_{\left\{\tau^{*}(x) \leqslant \sigma^{*}(y)\right\}}\right] \\
& +\mathbf{E}\left[e^{-q \sigma^{*}(y)}\left(e^{x \vee \bar{X}_{\sigma^{*}(y)}}-e^{y \vee \bar{X}_{\tau^{*}(x)}}\right) \mathbf{1}_{\left\{\sigma^{*}(y)<\tau^{*}(x),\right\}}\right] .
\end{aligned}
$$


Since for any $a$ it holds that $e^{x \vee a}-e^{y \vee a} \leqslant e^{x}-e^{y}$, we deduce

$$
V(x)-V(y) \leqslant e^{x}-e^{y} .
$$

Since $V\left(a^{*}\right)=e^{a^{*}}+\delta$ and since $V(x) \leqslant e^{x}+\delta$ for all $x$, it follows that $V(x)=e^{x}+\delta$ for all $x \in\left[0, a^{*}\right]$. The result follows.

4.2. The case $\delta \leqslant Z^{(q)}\left(y^{*}\right)-1$. Let us now conclude this section and the proof of Theorem 3 by establishing the following result. Recall that we are still under the regime $0<q<\psi(1)$.

Theorem 7. If $\delta \leqslant Z^{(q)}\left(y^{*}\right)-1$, then there exists a unique $z^{*} \in\left(0, y^{*}\right]$ which satisfies $Z^{(q)}\left(z^{*}\right)=1+\delta$. The value function is given by

$$
V(x)=e^{x} Z^{(q)}\left(z^{*}-x\right)
$$

and optimal stopping times are $\sigma^{*}=T_{0}^{-}$and $\tau^{*}=T_{z^{*}}^{+}$. In particular, part (ii) of Theorem 3 holds.

P r o of. Since $1+\delta \leqslant Z^{(q)}\left(y^{*}\right)$ and $Z^{(q)}(0)=1$, it follows that there exists a $z^{*} \in\left(0, y^{*}\right]$ such that $1+\delta=Z^{(q)}\left(z^{*}\right)$.

Next, note that from Lemma 2

$$
V(x)=e^{x} Z^{(q)}\left(z^{*}-x\right)=V\left(x, 0, z^{*}\right)
$$

and hence we can complete the proof by showing that the triple $\left(V, T_{z^{*}}^{+}, T_{0}^{-}\right)$ fulfils the conditions (i)-(vi) of Lemma 1 . It is immediately clear from the definition of $V$ that condition (i) holds. Next, note that $V^{\prime}(x)=e^{x} f\left(z^{*}-x\right)$. Since by Lemma 3 the function $f$ is strictly positive and since $V(0)=\delta$, it follows that $V(x) \leqslant \delta+e^{x}$ and hence condition (ii) of Lemma 1 holds. Conditions (iii) and (iv) are automatic.

To establish conditions (v) and (vi) of Lemma 1 one needs to appeal to the semimartingale decomposition of $e^{-\alpha t} V\left(Y_{t}^{x}\right)$. Similar arguments to those given in the proof of Proposition 1 lead to

$$
\begin{aligned}
e^{-\alpha t} V\left(Y_{t}^{x}\right) & =V(x)+\int_{0}^{t}(\Gamma-\alpha) V\left(Y_{s}^{x}\right) d s+\int_{0}^{t} V^{\prime}\left(Y_{s}^{x}\right) d\left(x \vee \bar{X}_{s}\right)+M_{t} \\
& =V(x)+\int_{0}^{t}(\Gamma-\alpha) V\left(Y_{s}^{x}\right) d s+V^{\prime}(0+)\left(x \vee \bar{X}_{t}\right)+M_{t}
\end{aligned}
$$

where $\Gamma$ is the generator of $-X$ under $\mathbf{P}^{1},(\Gamma-\alpha) V(x)=0$ for $x \in\left(0, z^{*}\right)$ and $(\Gamma-\alpha) V(x) \leqslant 0$ for $x>z^{*}$ and $M$ is a martingale. Note also that the term $V^{\prime}\left(Y_{s}^{x}\right)$ may be replaced by $V^{\prime}(0+)$ as $x \vee \bar{X}_{s}$ increases only when $Y_{s}^{x}=0$. From this, one sees in the above semimartingale decomposition that the process $\left\{e^{-\alpha t} V\left(Y_{t}^{x}\right): t \leqslant T_{0}^{-} \wedge T_{z^{*}}^{+}\right\}$is a martingale and that $\left\{e^{-\alpha t} V\left(Y_{t}^{x}\right): t \leqslant T_{0}^{-}\right\}$is a supermartingale. Again, right-continuity of paths is obvious. Since the second integral in (28) is equal to $V^{\prime}(0+)\left(x \vee \bar{X}_{t}\right)$ (in particular, it is an increasing, continuous, adapted process), it follows that the process $\left\{e^{-\alpha t} V\left(Y_{t}^{x}\right): t \leqslant T_{z^{*}}^{+}\right\}$is also a right-continuous submartingale. This completes the proof of Theorem 3 . 
5. Proof of Theorem 4. The proof goes along the lines of the proof of Theorem 7, principally appealing to the semimartingale decomposition (28) for the specified triple $\left(V, \tau^{*}, \sigma^{*}\right)$. For Theorem 4 (iv) it is possible to compute exactly the quantities $(\Gamma-\alpha) V(x)$ and $V^{\prime}(0+)$. In the remaining cases one may deduce the necessary properties of $(\Gamma-\alpha) V(x)$ as in the proof of Theorem 7 and that the process $V^{\prime}(0+)=f\left(z^{*} \wedge k^{*}\right) \geqslant 0$ from the properties of the function $f$ mentioned in Section 2 .

6. Proof of Theorem 2. Recall that for $q=0$, the pay-off of the game is given by

$$
G_{t, s}^{x}=e^{\bar{X}_{t} \vee x} \mathbf{1}_{\{t \leqslant s\}}+\left(e^{\bar{X}_{s} \vee x}+\delta e^{X_{s}}\right) \mathbf{1}_{\{s<t\}} .
$$

Lemma 5. Suppose that there exists some $\sigma^{*} \in \mathscr{T}_{0, \infty}$ such that

$$
\mathbf{E}\left[G_{\infty, \sigma^{*}}^{x}\right]=\inf _{\sigma \in \mathscr{T}_{0, \infty}} \mathbf{E}\left[G_{\infty, \sigma}^{x}\right]
$$

Then the Shepp-Shiryaev stochastic game (2) has value function $V$ and a saddle point is given by $\tau^{*}=\infty$ and $\sigma^{*}$. In other words, it is optimal for the inf-player to never stop.

P r o of. Let $\sigma \in \mathscr{T}_{0, \infty}$. Then it is straightforward to show that $G_{\bullet, \sigma}^{x}$ is a monotone nondecreasing function. We find that for any $\sigma, \tau \in \mathscr{T}_{0, \infty}$

$$
\mathbf{E}\left[G_{\tau, \sigma^{*}}^{x}\right] \leqslant \mathbf{E}\left[G_{\infty, \sigma^{*}}^{x}\right] \leqslant \mathbf{E}\left[G_{\infty, \sigma}^{x}\right]
$$

where the first inequality follows by the aforementioned monotonicity and the second inequality follows by the optimality of $\sigma^{*}$ in (30). Lemma 5 is proved.

$\mathrm{R}$ e $\mathrm{m} \mathrm{a} \mathrm{r} \mathrm{k} 3$ (problems with change of measure). It is tempting to solve (30) by the change of measure we have used throughout this paper, but the following example shows that when $q=0$, the corresponding optimal stopping problem under $\mathbf{P}^{1}$ is a different one.

Let $q=0$, and let $\psi(1)<0$ be such that $\psi^{\prime}(1) \geqslant 0$. Since $G_{s, t}^{x} \geqslant e^{x}$ for all $s, t$, we immediately see that $\inf _{\sigma \in \mathscr{T}_{0, \infty}} \mathbf{E}\left[G_{\infty, \sigma}^{x}\right] \geqslant e^{x}$. However, the optimal stopping problem under the changed measure is given by $\inf _{\sigma \in \mathscr{T}_{0, \infty}} \mathbf{E}\left[e^{\psi(1) \sigma}\left(e^{Y_{\sigma}^{x}}+\delta\right)\right]$. The latter optimal stopping problem has value zero, which can be seen by considering the sequence of stopping times $\left(\sigma_{n}\right)_{n \in \mathrm{N}}$ defined by $\sigma_{n}:=\inf \left\{t \geqslant n: Y_{t}^{x}=0\right\}$ which is almost surely finite under $\mathbf{P}^{1}$. The reason for this phenomenon is that the equality $\mathbf{E}\left[e^{x \vee \bar{X}_{\sigma}}+\right.$ $\left.\delta e^{X_{\sigma}}\right]=\mathbf{E}^{1}\left[e^{Y_{\sigma}^{x}}+\delta\right]$ holds whenever $\mathbf{P}^{1}\{\sigma<\infty\}=\mathbf{P}\{\sigma<\infty\}=1$. Since $X$ drifts to $-\infty$ under $\mathbf{P}$, we have that $\mathbf{P}\left\{\sigma_{n}<\infty\right\}<1$ for any $n \in \mathbf{N}$.

On account of the above remark, we consider (30) as an optimal stopping problem for $(\bar{X}, X)$, just as was done in the first paper on the Russian option [23]. We modify our notation and write for $y \geqslant x$

$$
V(x, y):=\inf _{\sigma \in \mathscr{T}_{0, \infty}} \mathbf{E}\left[e^{x \vee\left(y+\bar{X}_{\sigma}\right)}+\delta e^{y+X_{\sigma}}\right] .
$$


Again by standard theory on optimal stopping we know there exists a (possibly infinite) stopping time $\sigma^{*}=\sigma^{*}(x, y)$ at which the infimum in (31) is attained. We have the following verification lemma for (30), the proof of which is omitted, since it is similar to the proof of Lemma 1.

Lemma 6. Let $\sigma^{*} \in \mathscr{T}_{0, \infty}$ and let $V^{*}(x, y)=\mathbf{E}\left[e^{x \vee\left(y+\bar{X}_{\sigma^{*}}\right)}+\delta e^{y+X_{\sigma^{*}}}\right]$. Then $\left(V^{*}(x, 0), \sigma^{*}\right)$ is a solution to (30) if

(i) $V^{*}(x, y) \leqslant e^{x}+\delta e^{y}$,

(ii) the process $\left\{V^{*}\left(\bar{X}_{t}, X_{t}\right): t \geqslant 0\right\}$ is a right-continuous submartingale.

$\mathrm{P}$ r o of of $\mathrm{T}$ h e o r e $\mathrm{m} 2$. First, suppose $\psi(1) \geqslant 0$. Then the process $\left\{M_{t}\right\}_{t \geqslant 0}$ defined by $M_{t}=e^{x \vee\left(y+\bar{X}_{t}\right)}+\delta e^{y+X_{t}}$ is a $\mathbf{P}$-submartingale. Indeed, for $0 \leqslant s \leqslant t$ we have

$$
\begin{aligned}
\mathbf{E}\left[M_{t} \mid \mathscr{F}_{s}\right] & \geqslant e^{x \vee\left(\bar{X}_{s}+y\right)}+\delta e^{y+X_{s}} \mathbf{E}\left[e^{\widetilde{X}_{t-s}}\right] \\
& =e^{x \vee\left(\bar{X}_{s}+y\right)}+\delta e^{y+X_{s}} e^{\psi(1)(t-s)} \geqslant M_{s},
\end{aligned}
$$

where $\tilde{X}$ denotes an independent copy of $X$. Hence, in this case Lemma 6 shows that $V(x)=e^{x}+\delta$ and $\sigma^{*}=0$ solve (30), which agrees with part (i) of Theorem 2.

Next, let $\psi(1)<0$ and $\delta(\Phi(0)-1)>1$ and consider $\sigma^{*}=\infty$. Since $\bar{X}_{\infty}$ is exponentially distributed with parameter $\Phi(0)$, we find for $x \geqslant y$

$$
\begin{aligned}
V^{*}(x, y) & :=\mathbf{E}\left[e^{x \vee\left(y+\bar{X}_{\infty}\right)}+\delta e^{y+X_{\infty}}\right]=\mathbf{E}\left[e^{x \vee\left(\bar{X}_{\infty}+y\right)}\right] \\
& =e^{x} \int_{0}^{x-y} \Phi(0) e^{-\Phi(0) z} d z+\int_{x-y}^{\infty} \Phi(0) e^{-\Phi(0) z+z+y} d z \\
& =e^{x}\left(1-e^{-\Phi(0)(x-y)}\right)+\Phi(0)(\Phi(0)-1)^{-1} e^{x} e^{-\Phi(0)(x-y)} \\
& =e^{x}+(\Phi(0)-1)^{-1} e^{-(\Phi(0)-1) x+\Phi(0) y}
\end{aligned}
$$

and in particular, by the condition on $\delta$

$$
V^{*}(x, y) \leqslant e^{x}+\delta e^{y}
$$

Since $X$ is a strong Markov process, we have that $\bar{X}_{\infty}=\bar{X}_{t} \vee\left(X_{t}+\bar{X}_{\infty}^{\prime}\right)$, where $\bar{X}_{\infty}^{\prime}$ is a copy of $\bar{X}_{\infty}$ which is independent of $\mathscr{F}_{t}$ and thus

$$
\begin{aligned}
\mathbf{E}\left[e^{x \vee\left(\bar{X}_{\infty}+y\right)} \mid \mathscr{F}_{t}\right] & =\mathbf{E}\left[e^{x \vee\left(\bar{X}_{t}+y\right) \vee\left(X_{t}+y+\bar{X}_{\infty}^{\prime}\right)} \mid \mathscr{F}_{t}\right] \\
& =V^{*}\left(x \vee\left(\bar{X}_{t}+y\right), X_{t}+y\right) .
\end{aligned}
$$

It now follows that $\left\{V^{*}\left(\bar{X}_{t}, X_{t}\right)\right\}_{t \geqslant 0}$ is a $\mathbf{P}$-martingale (and hence in particular a submartingale). Again using Lemma 6 , we deduce part (ii) of Theorem 2.

Finally, let $\psi(1)<0$ and $\delta(\Phi(0)-1) \leqslant 1$ and take $x \geqslant y$. If we take

$$
\sigma^{*}=\tau_{x}^{+}=\inf \left\{t>0: X_{t} \geqslant x\right\}=T_{0}^{-},
$$


we have

$V^{*}(x, y):=\mathbf{E}\left[e^{\bar{X}_{\sigma^{*}}}+\delta e^{X_{\sigma^{*}}}\right]=e^{x}+\delta e^{x} \mathbf{P}\left\{\tau_{x-y}^{+}<\infty\right\}=e^{x}+\delta e^{-(\Phi(0)-1) x+\Phi(0) y}$ and again we have that $V^{*}(x, y) \leqslant e^{x}+\delta e^{y}$. Since $\left\{e^{\Phi(0) X_{t}}\right\}$ is a martingale, the submartingale property follows from Itô's formula and the fact that

$$
\begin{aligned}
\int_{0}^{t} \frac{\partial}{\partial x} V^{*}\left(\bar{X}_{t}, X_{t}\right) d \bar{X}_{t} & =\int_{0}^{t}\left(e^{\bar{X}_{t}}+\delta(1-\Phi(0)) e^{-\langle\Phi(0)-1) \bar{X}_{t}+\Phi(0) X_{t}}\right) d \bar{X}_{t} \\
& =\int_{0}^{t} e^{\bar{X}_{t}}(1+\delta-\Phi(0) \delta) d \bar{X}_{t} \geqslant 0
\end{aligned}
$$

where the second equality is due to the fact that $\bar{X}_{t}$ only increases when $X_{t}=\bar{X}_{t}$ and the proof of Theorem 2 is complete.

\section{Concluding remarks.}

$\mathrm{R}$ e $\mathrm{mark} 4$. In the proof of Theorem 6, the spectral negativity of the process is not used. This indicates that if a solution to the game exists, then the (possibly empty) sets $I_{1}, I_{2}$, and $I_{3}$ defined by

$$
\begin{aligned}
& I_{1}:=\left\{x \in[0, \infty): V(x)=e^{x}+\delta\right\} \\
& I_{2}:=\left\{x \in[0, \infty): e^{x}<V(x)<e^{x}+\delta\right\} \\
& I_{3}:=\left\{x \in[0, \infty): V(x)=e^{x}\right\}
\end{aligned}
$$

satisfy

$$
x_{1}<x_{2}<x_{3} \quad \text { for all } x_{i} \in I_{i}, i=1,2,3,
$$

and thus the solution to the game, if it exists, must be of the same nature for a more general Lévy process. An existence result for Nash (saddle point) equilibrium and the weaker Stackelberg equilibrium to optimal stopping games in a general Markovian setting (including Lévy processes) can be found in [9]. In that paper, optimal stopping games are considered with a pay-off function of the general form

$$
G_{1}\left(X_{\tau}\right) \mathbf{1}_{\{\tau<\sigma\}}+G_{1}\left(X_{\sigma}\right) \mathbf{1}_{\{\sigma<\tau\}}+G_{3}\left(X_{\tau}\right) \mathbf{1}_{\{\sigma=\tau\}},
$$

where $\tau$ and $\sigma$ are the strategies of the sup-player and inf-player, respectively.

For the Shepp-Shiryaev game the right-continuous, quasi-left-continuous strong Markov process is $\left(t, X_{t}, \bar{X}_{t}\right)$ and the functions $G_{1}, G_{2}$, and $G_{3}$ are given by $G_{1}(t, x, s)=G_{3}(t, x, s)=e^{-q t+s}$ and $G_{2}(t, x, s)=e^{-q t}\left(e^{s}+\delta e^{x}\right)$. The assumptions

$$
\mathbf{E}_{x} \sup _{t \geqslant 0}\left|G_{i}\left(X_{t}\right)\right|<\infty \quad \text { for } \quad x \in \mathbf{R} \quad(i=1,2,3)
$$

in [9] on the pay-off functions [to imply existence of a Nash equilibrium (saddle point) to the corresponding optimal stopping game] are consistent with the traditional assumption

$$
\mathbf{E}_{x} \sup _{t \geqslant 0}\left|G\left(X_{t}\right)\right|<\infty
$$


for results concerning existence of solutions to optimal stopping problems with pay-off $G$. We find that we cannot always fit the Shepp-Shiryaev game in this framework because of the same reason the Russian optimal stopping problem does not always satisfy the assumptions of traditional optimal stopping theorems. For example, when $X$ is a spectrally negative Lévy process with $\psi(1)>q$, the conditions (32) are not satisfied, since $\mathbf{E} \sup _{t \geqslant 0}\left|G_{2}\left(t, X_{t}, \bar{X}_{t}\right)\right|$ is bounded from below by $\sup _{t \geqslant 0} \delta \mathbf{E}\left[e^{-q t+X_{t}}\right] \geqslant$ $\sup _{t \geqslant 0} \delta e^{(\psi(1)-q) t}=\infty$. On the other hand, if we consider the SheppShiryaev game with finite horizon, then the assumptions in [9] are automatically satisfied thus guaranteeing a saddle point.

It should also be pointed out that there are examples of optimal stopping games which have a value but where the failure of (32) means that there is also failure for the existence of a saddle point. See, for example, [10].

$\mathrm{R}$ e m a r k 5. When $X$ is a Brownian motion with parameter $\sigma>0$ and drift $\mu$, it can be directly checked by taking Laplace transforms that the scale functions for $X$ are given by

$$
W^{(q)}(x)=2\left(\sigma^{2} \epsilon\right)^{-1} e^{\beta x} \operatorname{sh}(\epsilon x), \quad Z^{(q)}(x)=e^{\beta x} \operatorname{ch}(\epsilon x)-\epsilon^{-1} \beta e^{\gamma x} \operatorname{sh}(\epsilon x),
$$

where

$$
\epsilon=\sigma^{-1} \sqrt{\frac{\mu^{2}}{\sigma^{2}+2 q}} \text { and } \beta=-\frac{\mu}{\sigma^{2}} .
$$

In [15] the game is solved whenever $q>\psi(1) \geqslant 0$. Since, when $\delta$ is large enough, $z^{*}$ satisfies $Z^{(q)}\left(z^{*}\right)=1+\delta$, we find that $k_{*}:=e^{z^{*}}$ solves

$$
k_{*}^{\beta}\left(k_{*}^{\epsilon}+k_{*}^{-\epsilon}\right)-\epsilon^{-1} \beta k_{*}^{\beta}\left(k_{*}^{\epsilon}-k_{*}^{-\epsilon}\right)=2(1+\delta),
$$

which agrees with (7) in [15]. In [15] there is an additional technical condition (4) on the optimal stopping boundary $k_{*}$. The aforementioned condition pertains to the requirement that $V^{\prime}(0+) \geqslant 0$ (which ultimately is required for the appropriate submartingale property to hold). Working here with general spectrally negative Lévy processes, and in particular with scale functions, has seemingly produced arguments which have circumvented the need for such a condition. Hence, from the results in this paper, it follows that the claim in [15] (that this condition is necessary) is in fact false.

Acknowledgments. Part of this work was carried out whilst the first author was visiting the Department of Actuarial Mathematics and Statistics at Heriot-Watt University and the Department of Mathematical Sciences at The University of Bath. He is grateful for their hospitality. Both authors are grateful to Goran Peskir who, through numerous discussions, carefully explained the difference between Stackelberg equilibrium and Nash equilibrium as documented in [9] and [20]. Part of this research has been supported by EPSRC grant number EP/D045460/1. We are also grateful to an anonymous referee for the insightful remarks. 


\section{REFERENCES}

1. Alili L., Kyprianou A. E. Some remarks on first passage of Lévy processes, the American put and pasting principles. - Ann. Appl. Probab., 2005, v. 15, № 3, p. 2062-2080.

2. Avram F., Kyprianou A.E., Pistorius M.R. Exit problems for spectrally negative Lévy processes and applications to (Canadized) Russian options. - Ann. Appl. Probab., 2004, v. 14, № 1, p. 215-238.

3. Baurdoux E. J., Kyprianou A.E. The McKean stochastic game driven by a spectrally negative Lévy process. - Electron. J. Probab., 2008, v. 13, p. 173-197.

4. Bertoin J. Lévy Processes. Cambridge: Cambridge Univ. Press, 1996, 265 p.

5. Bichteler K. Stochastic Integration with Jumps. Cambridge: Cambridge Univ. Press, 2002, $501 \mathrm{p}$.

6. Duistermaat J. J., Kyprianou A. E., van Schaik K. Finite expiry Russian options. Stochastic Process. Appl., 2005, v. 115, № 4, p. 609-638.

7. Eisenbaum N., Kyprianou A.E. On the parabolic generator of a general onedimensional Lévy process. - Electron. Commun. Probab., 2008, v. 13, p. 198-209.

8. Ekström E. Russian options with a finite time horizon. - J. Appl. Probab., 2004, v. 41, № 2, p. 313-326.

9. Ekström E., Peskir G. Optimal stopping games for Markov processes. - SIAM J. Control Optim., 2008, v. 47, № 2, p. 684-702.

10. Ekström E., Villeneuve S. On the value of optimal stopping games. - Ann. Appl. Probab., 2006, v. 16, № 3, p. 1576-1596.

11. Helmes K., Stockbridge R. H. Linear programming approach to the optimal stopping of singular stochastic processes. - Stochastics, 2006, v. 79, № 3-4, p. 309-335.

12. Kifer Yu. Game options. - Finance Stoch., 2000, v. 4, № 4, p. 443-463.

13. Kühn C., Kyprianou A.E. Israeli options as composite exotic options. Preprint № 1298. Utrecht: Department of Mathematics, Utrecht University, 2004.

14. Kühn C., Kyprianou A.E. Callable puts as composite exotic options. - Math. Finance, 2007 , v. 17 , № 4, p. 487-502.

15. Kyprianou A. E. Some calculations for Israeli options. - Finance Stoch., 2004, v. 8, № 1, p. 73-86.

16. Kyprianou A.E. Introductory Lectures on Fluctuations of Lévy Processes with Applications. Berlin: Springer-Verlag, 2006, 373 p.

17. Kyprianou A.E., Surya B.A. A note on a change of variable formula with local time-space for Lévy processes of bounded variation. - Lecture Notes in Math., 2007, v. 1899 , p. $97-104$.

18. Peskir G. The Russian option: finite horizon. - Finance Stoch., 2005, v. 9, № 2, p. 251-267.

19. Peskir $G$. A change-of-variable formula with local time on surfaces. - Lecture Notes in Math., 2007, v. 1899, p. 69-96.

20. Peskir G. Optimal stopping games and Nash equilibrium. - Research Report № 28. Manchester: Probab. Statist. Group Manchester, 2007.

21. Peskir G., Shiryaev A.N. Optimal Stopping and Free-Boundary Problems. Basel: Birkhäuser, 2006, $500 \mathrm{p}$.

22. Protter $P$. Stochastic Integration and Differential Equations. Berlin: Springer-Verlag, 2004, $415 \mathrm{p}$.

23. Shepp L.A., Shiryaev A.N. The Russian option: reduced regret. - Ann. Appl. Probab., 1993, v. 3, № 3, p. 631-640.

24. Шепn Л. А., Ширяев А. Н. Новый взгляд на расчеты «Русского опциона». - Теория вероятн. и ее примен., 1994, т. 39, в. 1, с. 130-149.

Поступила в редакцию 18.VII. 2007

Исправленный вариант 16.IV.2008 